\title{
Clinicopathologic Correlates and Natural History of Atypical Chronic Myeloid Leukemia
}

2

* These authors contributed equally to this work.

Running Title: Features of Atypical Chronic Myeloid Leukemia

\section{Corresponding Author:}

Guillermo Montalban-Bravo, MD

Assistant Professor

The University of Texas MD Anderson Cancer Center

Department of Leukemia

1515 Holcombe Blvd, Unit 0428

Houston, TX 77030

Phone: +1-713-794-3604

Email: GMontalban1@mdanderson.org

Data Availability Statement: The datasets generated during and/or analyzed during the current study are not publicly available due to patient privacy concerns but are available from the corresponding author on reasonable request.

Key Words: Chronic Myeloid Leukemia, clinical outcomes, mutational codominance

Abstract Word Count: $192(200 \max )$

Text Word Count: 3405 (4 000 max)

References: 30 (60 max)

Tables: 3 ( $\max 8$ tables and figures)

\section{Figures: 4}

\section{Supplemental Files: 1}




\section{ABSTRACT}

There is limited data on the clonal mechanisms underlying leukemogenesis, prognostic factors,

3 and optimal therapy for atypical chronic myeloid leukemia (aCML). We evaluated the clinicopathological

4 features, outcomes, and responses to therapy of 65 patients with aCML. Median age was 67 years (range

5 46-89). The most frequently mutated genes included ASXL1 (83\%), SRSF2 (68\%), and SETBP1 (58\%).

6 Mutations in SETBP1, SRSF2, TET2, and GATA2 tended to appear within dominant clones, with frequent

7 SRSF2 and SETBP1 codominance, while other RAS pathway mutations were more likely to appear as

8 minor clones. Acquisition of new, previously undetectable mutations at transformation was observed in

$963 \%$ of evaluable patients, the most common involving signaling pathway mutations. Hypomethylating

10 agents were associated with the highest response rates and duration. With a median overall survival of

1125 months (95\% Cl 20-30), intensive chemotherapy was associated with worse OS than other treatment

12 modalities, and allogeneic stem cell transplantation was the only therapy associated with improved

13 outcomes (HR 0.044, 95\% Cl 0.035-0.593, $\mathrm{p}=0.007$ ). Age, platelet count, BM blast percentage, and

14 serum LDH levels were independent predictors of survival and were integrated in a multivariable model

15 which allowed to predict 1-year and 3-year survival.

16 


\section{INTRODUCTION}

2 suggests that co-mutation patterns may be associated with distinct MDS/MPN subtypes, with ASXL1 and

12 SETBP1 co-mutations frequently observed in aCML (7). However, the clonal dominance of identified 13 mutations in aCML remains poorly understood and the clonal mechanisms associated with disease 14 progression and transformation have not been well characterized. of transformation to acute myeloid leukemia $(\mathrm{AML})$ compared to other $\operatorname{MDS} / \operatorname{MPNs}(3,11)$, however there

17 is scarce data evaluating the potential factors associated with aCML prognosis (3). To date, therapeutic 18 options for patients with aCML are limited and, although therapy with ruxolitnib can be associated with 19 responses in patients with $\operatorname{CSF3R}$ mutations $(5,12,13)$, particularly in the absence of SETBP1 20 mutations, there is insufficient evidence on the optimal therapeutic strategies for these patients. Although

21 several reports including small patient cohorts have described the potential use of hypomethylating

22 agents, such as decitabine or azacitidine, for the treatment of aCML $(3,14-18)$, evaluation of the survival 23 benefit or clinical activity of these compounds a lager cohort and comparison to other therapeutic 24 approaches is needed. 
In order to study the clonal architecture and clinical outcomes of patients with aCML based on

2 therapeutics modality and factors associated with transformation and predictors of outcome, we evaluated

3 a cohort of 65 patients with aCML treated at a single institution.

\section{MATERIALS AND METHODS}

7 Patients and Samples

We evaluated all consecutive patients with atypical chronic myeloid leukemia treated at The

9 University of Texas MD Anderson Cancer Center (MDACC) from 2005 to 2020. Informed consent was

10 obtained according to protocols approved by the MDACC institutional review board in accordance with

11 the Declaration of Helsinki. Diagnosis of aCML was confirmed in a hematopathology laboratory at

12 MDACC using the 2016 WHO criteria, by two independent hematopathologists (CBR and RKS) (19).

13 Conventional karyotyping was performed on fresh BM aspirates using standard procedures and reported

14 following ISCN 2013 Nomenclature (20).

\section{Targeted gene sequencing analysis}

Genomic DNA was extracted from whole bone marrow aspirate samples and was subject to

18 targeted PCR-based sequencing using a NGS platform evaluating a total of 81 or 28 genes (21). This

19 analysis was performed within the MDACC CLIA-certified Molecular Diagnostics Laboratory (additional

20 details in Supplemental Methods). For NGS-based analysis, the limit of detection for variant calling was

$212 \%$. Previously described somatic mutations registered at the Catalogue of Somatic Mutations in Cancer

22 (COSMIC: http://cancer.sanger.ac.uk/cosmic) were considered as potential driver mutations. Variant

23 allele frequency (VAF) estimates of identified mutations were used to evaluate clonal relationships within

24 each individual patient, with clones with the highest VAF or with VAF close to 0.4 being defined as 
1 dominant and those present at $\mathrm{VAF}<0.2$ in the presence of another dominant clone being defined as

2 minor. In addition, PCR-based DNA analysis was performed to detect internal tandem duplications and

3 codon 835/836 point mutations in FLT3. Multiplex PCR using fluorescently labeled primers was

4 performed, followed by detection and sizing of PCR products using capillary electrophoresis. For

5 detecting point mutations in codons 835/836, restriction enzyme digestion of the PCR products was

6 performed prior to capillary electrophoresis. The lower limit of detection (analytical sensitivity) of this

7 assay was $1 \%$ of mutant DNA in a background of wild-type DNA. The ratio of the area under the peak of

8 mutant over total (mutant : wild-type) FLT3 was used to determine the mutant allele burden.

\section{Statistical analysis and response assessment}

VAF estimates were used to evaluate clonal relationships within each individual sample (22).

12 Clonal relationships were tested using Pearson goodness-of-fit tests with heterogeneity being defined in

13 cases with goodness-of-fit $p$-values $<0.05$. Response outcomes were evaluated following the MDS/MPN

14 IWG response criteria (23) for therapy at the time of aCML diagnosis and following ELN 2017 criteria for

15 therapies at the time of transformation to AML (24). Generalized linear models were used to study the

16 association of overall response (ORR), complete remission (CR), and risk factors. Overall survival (OS)

17 was calculated as the time from diagnosis to death or last follow-up date. Event-free survival (EFS) was

18 calculated from the time of initial therapy until relapse, absence of response, or death. Leukemia-free

19 survival (LFS) was calculated from the time of diagnosis to transformation, death, or last follow-up date.

20 Patients who were alive at their last follow-up were censored on that date. The Kaplan-Meier product limit

21 method (25) was used to estimate the median OS, EFS, and LFS for each clinical/demographic factor.

22 Univariate and multivariate Cox proportional hazards regression analyses were used to identify any

23 association with each of the variables and survival outcomes. 


\section{RESULTS}

\section{Clinical and Histopathological Characteristics}

A total of 65 patients with aCML were evaluated during the reviewed time period. Patient

5 characteristics are summarized in Table 1. Median age was 67 years (range 46-89), and median WBC

6 was $44.5 \times 10^{9} / \mathrm{L}$ (range $5.9-474.9 \times 10^{9} / \mathrm{L}$ ). Median percentages of immature granulocytes in peripheral

7 blood were as follows: 0\% (range 0-27\%) promyelocytes, $0 \%$ myelocytes (range 0-35\%), and $16 \%$

8 metamyelocytes (range 0-51\%). Median hemoglobin was $10.0 \mathrm{~g} / \mathrm{dL}$ (range $5.7-14.7 \mathrm{~g} / \mathrm{dL}$ ) and median

9 platelet count was $93 \times 10^{9} / \mathrm{L}$ (range $12-560 \times 10^{9} / \mathrm{L}$ ). Forty-one $(63 \%)$ patients had normal karyotype, with

10 the most frequent recurrent cytogenetic abnormalities including trisomy 8 in $5(8 \%)$ patients, $i(17 q)$ in 2

$11(3 \%)$ patients, and del(20q) in $2(3 \%)$ patients. A total of $3(5 \%)$ patients had complex karyotype defined

12 by presence of more than 3 abnormalities, but only 1 patient had a monosomal karyotype. Median

13 European Cooperative Group performance status at the time of diagnosis was 1 (range 0-4). 21 (32\%)

14 patients required transfusions prior to the time of evaluation. Significant palpable splenomegaly was

15 observed in $26(40 \%)$ patients and a total of $7(11 \%)$ had extramedullary disease, either confirmed by

16 histopathological evaluation or highly suspected due to imaging including: pathology proven leukemia

17 cutis in 3, gingival hyperplasia in 1 patient, and lymphadenopathy in 3 patients. Nineteen (29\%) patients

18 had required hydroxyurea for control of leukocytosis prior to their presentation at MDACC, with 6 (\%)

19 patients presenting with signs of spontaneous tumor lysis syndrome or acute renal dysfunction. Of these,

203 required rapid cytoreduction with cytarabine and 1 required leukophoresis. A total of $51(80 \%)$ received

21 therapy at MDACC, including single agent hypomethylating agent in $19(29 \%)$, hydroxyurea in $8(12 \%)$, a

22 hypomethylating agent in combination with ruxolitinib in $7(11 \%)$, single agent ruxolitinib in $5(8 \%)$,

23 hypomethylating agents in combination with other investigational agents in 5 (8\%), induction 
1 chemotherapy in $3(5 \%)$, and other investigational agents in $1(2 \%)$ patient. Two patients remained on

2 observation, 1 patient received allogeneic stem cell transplant directly, and 14 (22\%) patients continued

3 care outside of MDACC.

$4 \quad$ Bone marrow evaluation revealed a markedly hypercellular marrow in all patients with

5 granulocytic proliferation and granulocytic dysplasia. Significant dyserythropoiesis was observed in 26

$6(40 \%)$ patients with dysmegakaryopoiesis being observed in $43(66 \%)$ patients. Marked trilineage

7 dysplasia was apparent in $22(33 \%)$ patients. Bone marrow grading of fibrosis was performed on a total of

$852(80 \%)$ patients with 7 (13\%) patients having MF-0, 32 (62\%) MF-1, 11 (21\%) MF-2, and 2 (4\%) MF-3.

9 Median bone myeloid population frequencies are detailed in Table 2.

\section{Mutation and clonal landscape and clinicopathological associations}

12 Next-generation sequencing data was available for $35(54 \%)$ patients. The median number of

13 detectable mutations was 4 (range 1-8). The most frequently mutated genes included $A S X L 1$ in $83 \%$,

14 SRSF2 in $68 \%$, and SETBP1 in $58 \%$. The frequencies of identified mutations are shown in Figure $1 \mathrm{~A}$.

15 Mutations in ASXL1 included frameshift $(n=25)$ or nonsense $(n=4)$ mutations, the most common of which

16 being G646fs in 17/29 patients. The most frequent SETBP1 mutation included D868N. Other genes with

17 mutations present at a frequency $>10 \%$ of the evaluated population included TET2, CBL, GATA2, NRAS,

$18 R U N X 1, N F 1$, and EZH2. The median variant allele frequencies of identified mutations are shown in

19 Figure 1B.

20 In order to determine the likely clonal dominance of identified mutations, VAF estimates were

21 used to evaluate clonal relationships within each individual sample (22) using Pearson goodness-of-fit

22 tests and VAF differences. Clones with the highest VAFs or with VAFs close to $40 \%$ were defined as

23 dominant, and those present at VAF $<20 \%$ in the presence of another dominant clone were defined as 
minor. Mutations in SETBP1, SRSF2, TET2, and GATA2 tended to appear within dominant clones while other RAS pathway mutations were more likely to appear as minor clones. Within the observed commonly co-mutated genes, SRSF2 and SETBP1 tended to appear as co-dominant, while ASXL1, although the most frequently detected mutation, appeared as a minor clone in up to $50 \%$ of patients (Figure $1 \mathrm{C}$ ).

Cytogenetic and clonal evolution associated with transformation to acute myeloid leukemia months (1-123 months). Peripheral blood and bone marrow findings at the time of transformation are detailed in Supplemental Table S1.

11 sequencing at diagnosis of $\mathrm{aCML}$ and $\mathrm{AML}$ in $8(44 \%)$ patients. The mutational landscape at the time of

12 transformation is shown in Figure 2A. Acquisition of new, previously undetectable mutations was

13 observed in 5 patients, the most common involving signaling pathway mutations (NRAS, KRAS, NF1,

14 PTPN11) as well as FLT3-ITD, ASXL1, CEBPA, and ETV6 (Figure 2A). Acquisition of new cytogenetic

15 abnormalities was observed in 9/14 patients, the most frequent involving $\mathrm{i}(17 \mathrm{q})$. Dynamic changes in the

16 clonal and cytogenetic landscape and disease phenotype during the course of therapy from diagnosis to

17 transformation are shown in Figure 2B.

Clinical outcomes based on therapy type and genomic and clinical characteristics

21 who received disease-modifying agents were evaluable for response. Patients who continued observation

22 or cytoreductive therapy with hydroxyurea were not considered evaluable for response. Among response-

23 evaluable patients, $19(50 \%)$ received a single agent HMA, 6 (16\%) an HMA in combination with 
1 ruxolitinib, $5(13 \%)$ single agent ruxolitinib, $4(11 \%)$ an HMA in combination with other investigational

2 agents, $3(8 \%)$ induction chemotherapy, and $1(3 \%)$ proceeded directly to allogeneic stem -cell

3 transplantation. The ORR was $29 \%$, with a total of $3(8 \%)$ patients achieving CR. Response rates and

4 median response durations based on therapeutic modality are detailed in Table 2. Among patients who

5 received ruxolitinib, either as single agent or in combination with an HMA, 2 (17\%) had detectable JAK2

6 V617F mutations, and no patients had detectable CSF3R mutations.

7 The median OS of the entire cohort was 25 months (95\% Cl 20.0-30.0, Figure 3A). When

8 evaluating survival based on therapeutic regimen, patients who received intensive chemotherapy had

9 significantly worse OS than those receiving HMA-based therapy or other agents such as ruxolitinib or

10 hydroxyurea ( $p=0.012$, Figure $3 B$ ). Of note, among the 3 patients treated with intensive chemotherapy, 1

11 presented with an ECOG performance status of $4, \mathrm{WBC}$ of $207 \times 10^{9} / \mathrm{L}$, and spontaneous tumor lysis

12 syndrome with acute renal dysfunction at the time of diagnosis, 1 had leukemia cutis with a WBC of

$1325.8 \times 10^{9} / \mathrm{L}$, and one had gingival hyperplasia and a WBC of $181.4 \times 10^{9} / \mathrm{L}$. No significant differences in

14 survival were observed between patients receiving hydroxyurea, ruxolitinib, or an HMA alone or with other

15 agents. A total of 7 (11\%) patients underwent allogeneic stem cell transplantation. The median LFS was

1619.8 months (95\% Cl 15.6-24 months, Figure $3 \mathrm{C})$ and the median survival after transformation was 8.3

17 months (95\% CI 5.5-11.0 months). After transformation to AML 11 patients received therapy with an ORR

18 of $64 \%$, including $4(36 \%)$ CRis and a CR rate of $18 \%$. Therapies included: cladribine or clofarabine in

19 combination with low dose cytarabine (LDAC) in 3 patients; LDAC in combination with venetoclax in 1; an

20 HMA in combination with ruxolitinib in 1; an HMA in combination with venetoclax in 1; an HMA in

21 combination with other agents in 1; intensive chemotherapy with sorafenib in 1; investigational agents in

221 ; and myeloablative conditioning and transplant in 1 . The median number of cycles of therapy was 2

23 (range 1-5) with a median number of cycles to best response of 2 (range 1-5). Median response duration 
1 was 1.4 months (range $0-4$ ) and 2 patients were able to transition to allogeneic stem cell transplant.

2 Among patients who suffered transformation to AML, only 1 remains alive at the time of data cutoff and

3 analysis.

4 By univariate analysis for survival, peripheral blood promyelocyte percentage $(p=0.005)$,

5 performance status $\geq 2(p=0.059)$, hemoglobin $(p=0.033)$, bone marrow blast percentage $(p=0.013)$, and

6 bone marrow monocyte percentage $(p=0.027)$ were associated with survival (Supplemental Table S2). By

7 multivariate analysis for overall survival, age (HR 1.107, 95\% Cl 1.045-1.173, $\mathrm{p}=0.001$ ), hemoglobin (HR

$8 \quad 0.784,95 \%$ Cl $0.635-0.968, p=0.024)$, platelet count (HR 0.993, 95\% Cl 0.988-0.997, p=0.003), bone

9 marrow blast percentage (HR 1.414, 95\% Cl 1.223-1.635, $\mathrm{p}<0.001)$, bone marrow monocyte percentage

10 (HR 1.215, 95\% Cl 1.008-1.466, $\mathrm{p}=0.041)$, LDH levels (HR 1.000, 95\% Cl 1.000-1.000, $\mathrm{p}<0.001)$, and

11 allogeneic stem -cell transplantion ( $\mathrm{HR} 0.044,95 \% \mathrm{Cl} 0.035-0.593, \mathrm{p}=0.007$ ) were independent predictors

12 of survival (Supplemental Table S2). In order to evaluate disease and patient related features that could

13 allow prediction of the clinical outcomes of patients with aCML at the time of diagnosis, we performed

14 multivariate analysis for survival based on baseline clinicopathological features. The following patient

15 characteristics were independently associated with patient prognosis (Table 3): age, platelet count, bone

16 marrow blast percentage, and LDH levels. This model was used to generate a nomogram for overall

17 survival (Figure 4). This nomogram provides a visual depiction of the relative contribution of each

18 prognostic factor to the total point score and the weight of factors influencing survival. The formula for

19 calculating the total point score is as follows: age points $(+60.18185+1.337374 \times$ age $)+$ platelet points

$20(59.82308+-0.99705 \times$ platelet count $)+$ bone marrow blast points $(4.54877 \times$ bone marrow blast \%) +

21 LDH points $(0.002500 \times \mathrm{LDH}$ level). Total point scores ranged from 36.1 to 165.1 , with a median of 95.0.

22

\section{DISCUSSION}


Atypical chronic myeloid leukemia $(\mathrm{aCML})$ is a rare hematopoietic stem cell disorder with dismal

2 prognosis and a high rate of transformation to acute leukemia (11). Although prior reports have described

3 activity of ruxolitinib (12), hydroxyurea, low-dose cytarabine, or HMAs $(15,16,26)$ in this disease, data on

4 the optimal clinical management of these patients remains unclear. In addition, given the rarity of this

5 disorder, there is a lack of validated clinical risk models to effectively stratify patients based on predicted

6 outcomes $(3,26)$. Finally, although several studies have described recurrent somatic mutations in aCML,

7 the clonal architecture in aCML and the genomic changes associated with transformation remain unclear.

8 In this study, we evaluated the clinicopathological features, outcomes, and clonal architecture of a cohort

9 of 65 patients with aCML. By doing so, we observed a high frequency of SRSF2 and SETBP1 mutation

10 co-dominance, with ASXL1 mutations being the most frequently observed; acquisition or clonal expansion

11 of previously undetected RAS pathway mutations; and certain cytogenetic abnormalities, such as i(17q)

12 or monosomy 7 , associated with acute transformation. This is consistent with prior reports by our group

13 associating $\mathrm{i}(17 \mathrm{q})$ with transformation to $\mathrm{AML}$ in MDS/MPNs (27). Finally, we developed a prognostic

14 model which included age, platelet count, bone marrow blast percentage, and LDH, which allowed us to

15 predict the survival of patients with aCML.

17 recently published study by Palomo, et al (7), mutations in ASXL1 strongly correlated with SETBP1

18 mutations in patients with aCML. Although in this study the authors identified ASXL1 mutations as part of

19 ancestral clones in a majority of patients (79\%), we identified that both SRSF2 and SETBP1 mutations

20 tended to appear at significantly higher VAFs and as dominant events in a majority of patients, while

21 ASXL1 mutations appeared in minor clones in up to $50 \%$ of patients. In addition, similar to their findings,

22 we observed that GATA2 mutations tended to appear within dominant clones and RAS pathway signaling

23 gene mutations tended to appear in minor clones. However, sequential targeted sequencing performed at 
1 the time of transformation revealed that, although initially present within non-dominant clones, mutations

2 in signaling genes other than SETBP1 were associated with clonal expansion and transformation to AML.

3 This data suggests that, although other RAS pathway signaling mutations might be less common and

4 appear as subclonal events in aCML, their expansion through the course of therapy or their acquisition in

5 initial founder clones may likely be responsible for transformation and resistance to therapies. This might

6 be relevant when considering future therapeutic combinations with agents such as BCL2 inhibitors, given

7 the known association of these mutations with resistance to therapies such as venetoclax (28). In

8 addition, this underscores the importance of developing effective agents targeting RAS signaling or MCL-

91 in the current era of venetoclax-based therapies $(29,30)$.

10 Although prior reports have suggested that therapy with single agent ruxolitinib might be effective

11 in patients with aCML, we did not observe significant responses. However, none of the 5 patients who

12 received single agent ruxolitinib in our cohort had CSF3R mutations and only 2 had JAK2 mutations. In

13 addition, the combination of azacitidine with ruxolitinib was not associated with any significant responses.

14 Prior case reports described the potential efficacy of azacitidine or decitabine in patients with aCML. In

15 our study, response rates to HMA-based therapies were observed in $25 \%$ of patients, and only 3 patients

16 achieved CR, with a median response duration of 2.7 months. Although use of intensive chemotherapy

17 was associated with worse survival, patients treated with this therapeutic modality had highly proliferative

18 disease with extramedullary involvement and spontaneous tumor lysis, suggesting that their underlying

19 disease biology was likely responsible for the shorter survival times. Allogeneic stem cell transplantation

20 was the only therapeutic strategy that was associated with significantly improved survival, suggesting that

21 all patients who are eligible should be considered for transplant.

22 Prior studies evaluating a cohort of 55 and 25 patients with aCML reported advanced age, high

23 WBC, low hemoglobin, presence of immature granulocytic precursors, and TET2 mutations to be 
1 associated with worse survival in patients with $\operatorname{aCML}(3,26)$. In our study, with one of the largest cohorts

2 so far reported, blood immature granulocyte percentage and bone marrow monocytosis were associated

3 with worse survival by univariate analysis, but lost their independent prognostic significance in

4 multivariate analysis, while age, platelet count, bone marrow blast percentage, and LDH levels remained

5 independent predictors of survival. Integration of these variables into a multivariate Cox regression model

6 allowed us to create a nomogram that predicted 1-year and 3-year overall survival. Although further

7 validation of this model is warranted, its integration into clinical practice may allow more specific survival

8 estimates when compared to conventional cutoff driven scoring systems.

9 We acknowledge that our study has several limitations. First, its retrospective nature limits our

10 ability to unequivocally confirm survival and response differences based on distinct therapeutic

11 interventions. Second, the absence of NGS in all evaluated patients limited our ability to incorporate

12 somatic mutation data as part of the multivariate prognostic model, and only a subset of patients who

13 transformed to AML had sequencing at the time of progression. Finally, although this study includes one

14 of the largest reported clinically annotated cohorts of patients with aCML, prospective studies will be

15 necessary to confirm the optimal therapeutic modality for patients with aCML.

16 Despite these limitations, our data suggest that $\mathrm{aCML}$ is characterized by specific mutational

17 clonal dominance with a high frequency of co-dominant SRSF2 and SETBP1 mutations, and other RAS

18 pathway mutations present in minor clones at the time of diagnosis but associated with AML

19 transformation. In addition, we observed that acquisition of $\mathrm{i}(17 \mathrm{q})$ is associated with $\mathrm{AML}$ transformation.

20 Also, we confirm poor survival and response outcomes with most treatment modalities, with HMA

21 treatment associated with the highest and most durable responses. Finally, incorporation of age, platelet

22 count, bone marrow blast percentage, and LDH levels can allow survival prediction for these patients, and 
1 allogeneic stem cell transplantation should be considered on all eligible patients with a diagnosis of 2 aCML.

3

4 ACKNOWLEDGEMENTS: This work was supported in part by the University of Texas MD Anderson

5 Cancer Center Support Grant CA016672 and the University of Texas MD Anderson Cancer Center

6 MDS/AML Moon Shot.

7

8 CONFLICTS OF INTEREST: The authors declare no competing interests.

9

10

11 


\section{REFERENCES}

2 1. Arber DA, Orazi A, Hasserjian R, Thiele J, Borowitz MJ, Le Beau MM, et al. The 2016 revision to the

3 World Health Organization classification of myeloid neoplasms and acute leukemia. Blood. $4 \quad 2016 ; 127(20): 2391-405$.

5 2. Makishima H, Yoshida K, Nguyen N, Przychodzen B, Sanada M, Okuno Y, et al. Somatic SETBP1 $6 \quad$ mutations in myeloid malignancies. Nature genetics. 2013;45(8):942-6.

7 3. Patnaik MM, Barraco D, Lasho TL, Finke CM, Reichard K, Hoversten KP, et al. Targeted next

4. Langabeer SE, Comerford CM, Quinn J, Murphy PT. Molecular profiling and targeted inhibitor therapy in atypical chronic myeloid leukaemia in blast crisis. J Clin Pathol. 2017;70(12):1089.

12 5. Ammatuna E, Eefting M, van Lom K, Kavelaars FG, Valk PJ, Touw IP. Atypical chronic myeloid leukemia with concomitant CSF3R T618I and SETBP1 mutations unresponsive to the JAK inhibitor ruxolitinib. Ann Hematol. 2015;94(5):879-80.

6. Gambacorti-Passerini CB, Donadoni C, Parmiani A, Pirola A, Redaelli S, Signore G, et al. Recurrent ETNK1 mutations in atypical chronic myeloid leukemia. Blood. 2015;125(3):499-503.

7. Palomo L, Meggendorfer M, Hutter S, Twardziok S, Adema V, Fuhrmann I, et al. Molecular landscape and clonal architecture of adult myelodysplastic/myeloproliferative neoplasms. Blood. 2020.

8. Maxson JE, Gotlib J, Pollyea DA, Fleischman AG, Agarwal A, Eide CA, et al. Oncogenic CSF3R mutations in chronic neutrophilic leukemia and atypical CML. N Engl J Med. 2013;368(19):1781-90.

9. Yun JW, Yoon J, Jung CW, Lee KO, Kim JW, Kim SH, et al. Next-generation sequencing reveals unique combination of mutations in cis of CSF3R in atypical chronic myeloid leukemia. J Clin Lab Anal. 2020;34(2):e23064.

10. Zhang H, Wilmot B, Bottomly D, Dao KT, Stevens E, Eide CA, et al. Genomic landscape of neutrophilic leukemias of ambiguous diagnosis. Blood. 2019;134(11):867-79. myeloid leukemia is clinically distinct from unclassifiable myelodysplastic/myeloproliferative neoplasms. Blood. 2014;123(17):2645-51. 
12. Dao KT, Gotlib J, Deininger MMN, Oh ST, Cortes JE, Collins RH, Jr., et al. Efficacy of Ruxolitinib in Patients With Chronic Neutrophilic Leukemia and Atypical Chronic Myeloid Leukemia. J Clin Oncol. 2020;38(10):1006-18.

13. Dao KH, Solti MB, Maxson JE, Winton EF, Press RD, Druker BJ, et al. Significant clinical response to JAK1/2 inhibition in a patient with CSF3R-T618I-positive atypical chronic myeloid leukemia. Leuk Res Rep. 2014;3(2):67-9.

14. Andriani A, Elli E, Trape G, Villiva N, Fianchi L, Di Veroli A, et al. Treatment of Philadelphia-negative myeloproliferative neoplasms in accelerated/blastic phase with azacytidine. Clinical results and identification of prognostic factors. Hematol Oncol. 2019;37(3):291-5.

15. Andriani A, Montanaro M, Voso MT, Villiva N, Ciccone F, Andrizzi C, et al. Azacytidine for the treatment of retrospective analysis from the Gruppo Laziale for the study of Ph-negative MPN. Leuk Res. 2015;39(8):801-4.

16. Hausmann H, Bhatt VR, Yuan J, Maness LJ, Ganti AK. Activity of single-agent decitabine in atypical chronic myeloid leukemia. J Oncol Pharm Pract. 2016;22(6):790-4.

17. Marumo A, Mizuki T, Tanosaki S. Atypical chronic myeloid leukemia achieving good response with azacitidine. Indian J Cancer. 2019;56(4):354-5.

18. Tong X, Li J, Zhou Z, Zheng D, Liu J, Su C. Efficacy and side-effects of decitabine in treatment of atypical chronic myeloid leukemia. Leukemia \& lymphoma. 2015;56(6):1911-3.

19. Arber DA, Orazi A, Hasserjian R, Thiele J, Borowitz MJ, Le Beau MM, et al. The 2016 revision to the World Health Organization (WHO) classification of myeloid neoplasms and acute leukemia. Blood. 2016.

20. Simons A, Shaffer LG, Hastings RJ. Cytogenetic Nomenclature: Changes in the ISCN 2013 Compared to the 2009 Edition. Cytogenet Genome Res. 2013;141(1):1-6.

21. Lindsley RC, Mar BG, Mazzola E, Grauman PV, Shareef S, Allen SL, et al. Acute myeloid leukemia ontogeny is defined by distinct somatic mutations. Blood. 2015;125(9):1367-76.

22. Papaemmanuil E, Gerstung M, Malcovati L, Tauro S, Gundem G, Van Loo P, et al. Clinical and biological implications of driver mutations in myelodysplastic syndromes. Blood. 2013;122(22):361627; quiz 99. 
23. Savona MR, Malcovati L, Komrokji R, Tiu RV, Mughal TI, Orazi A, et al. An international consortium proposal of uniform response criteria for myelodysplastic/myeloproliferative neoplasms (MDS/MPN) in adults. Blood. 2015;125(12):1857-65.

24. Dohner H, Estey E, Grimwade D, Amadori S, Appelbaum FR, Buchner T, et al. Diagnosis and management of AML in adults: 2017 ELN recommendations from an international expert panel. Blood. 2017;129(4):424-47.

25. Kaplan EI MP. Nonparametric estimation from incomplete observations. Journal of the American Statistical Association. 1958(53):457-81.

26. Breccia M, Biondo F, Latagliata R, Carmosino I, Mandelli F, Alimena G. Identification of risk factors in atypical chronic myeloid leukemia. Haematologica. 2006;91(11):1566-8.

27. Kanagal-Shamanna R, Bueso-Ramos CE, Barkoh B, Lu G, Wang S, Garcia-Manero G, et al. Myeloid neoplasms with isolated isochromosome 17q represent a clinicopathologic entity associated with myelodysplastic/myeloproliferative features, a high risk of leukemic transformation, and wild-type TP53. Cancer. 2012;118(11):2879-88.

28. DiNardo CD, Tiong IS, Quaglieri A, MacRaild S, Loghavi S, Brown FC, et al. Molecular patterns of response and treatment failure after frontline venetoclax combinations in older patients with AML. Blood. 2020;135(11):791-803.

29. Hormi M, Birsen R, Belhadj M, Huynh T, Cantero Aguilar L, Grignano E, et al. Pairing MCL-1 inhibition with venetoclax improves therapeutic efficiency of BH3-mimetics in AML. Eur J Haematol. 2020.

30. Tambe M, Karjalainen E, Vaha-Koskela M, Bulanova D, Gjertsen BT, Kontro M, et al. Pan-RAF inhibition induces apoptosis in acute myeloid leukemia cells and synergizes with BCL2 inhibition. Leukemia. 2020. 


\section{TABLES}

\section{Table 1. Patient Characteristics}

\begin{tabular}{|c|c|}
\hline \multirow[t]{2}{*}{ Characteristic } & aCML (N=65) \\
\hline & N (\%)/Median [range] \\
\hline Age (years) & 68 [46-89] \\
\hline Male & $45(69)$ \\
\hline WBC $\left(\times 10^{9} / L\right)$ & $44.5[5.9-474.9]$ \\
\hline Neutrophils (\%) & $64[0-93]$ \\
\hline Promyelocytes (\%) & $0[0-27]$ \\
\hline Myelocytes (\%) & $0[0-66]$ \\
\hline Metamyelocytes (\%) & $16[0-51]$ \\
\hline Lymphocytes (\%) & 8 [0-63] \\
\hline Monocytes (\%) & $2[0-13]$ \\
\hline Basophil (\%) & $0[0-6]$ \\
\hline Eosinophil (\%) & $1[0-15]$ \\
\hline Blast (\%) & $1[0-16]$ \\
\hline $\mathrm{Hgb}(\mathrm{g} / \mathrm{dL})$ & $10[5.7-14.7]$ \\
\hline Platelets $\left(\times 10^{9} / \mathrm{L}\right)$ & 93 [12-560] \\
\hline BM blast (\%) & $2[0-16]$ \\
\hline BM progranulocytes (\%) & $1[0-12]$ \\
\hline BM myelocytes (\%) & $16[2-41]$ \\
\hline BM metamyelocytes (\%) & 5 [5-31] \\
\hline BM granulocytes (\%) & $36[10-77]$ \\
\hline BM basophils (\%) & 0 [0-20] \\
\hline BM eosinophils (\%) & $1[0-11]$ \\
\hline BM monocytes (\%) & $2[0-10]$ \\
\hline \multicolumn{2}{|l|}{ Cytogenetics } \\
\hline Normal & $41(63)$ \\
\hline Trisomy 8 & $5(8)$ \\
\hline $\mathrm{i}(17 \mathrm{q})$ & $2(3)$ \\
\hline Monosomy $\mathrm{Y}$ & $1(2)$ \\
\hline $\operatorname{Del}(20 q)$ & $2(3)$ \\
\hline Complex & $3(5)$ \\
\hline Other & $9(14)$ \\
\hline NA & $2(3)$ \\
\hline
\end{tabular}




\begin{tabular}{|l|c|}
\hline Therapy related (\%) & $1(2)$ \\
\hline ECOG Performance status & $45(69)$ \\
\hline $0-1$ & $12(18)$ \\
\hline$\geq 2$ & $21(32)$ \\
\hline Prior transfusions & $26(40)$ \\
\hline Splenomegaly & $7(11)$ \\
\hline Extramedullary disease & $16(25)$ \\
\hline B symptoms & \\
\hline
\end{tabular}

2 Table 2. Response outcomes in patients with aCML based on therapy

\begin{tabular}{|c|c|c|c|c|c|}
\hline Response & $\begin{array}{c}\text { HMA } \\
(n=19) \\
N(\%) /[\text { range] }\end{array}$ & $\begin{array}{c}\text { HMA+ruxolitin } \\
\text { ib } \\
(n=6) \\
N(\%) /[\text { range] }\end{array}$ & 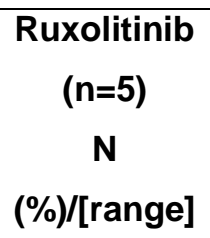 & $\begin{array}{l}\text { HMA-combo } \\
(n=4) \\
N(\%) /[\text { range }]\end{array}$ & $\begin{array}{c}\text { Chemotherapy } \\
\qquad \begin{array}{c}(n=3) \\
N(\%) /[\text { range] }\end{array}\end{array}$ \\
\hline Overall response rate & $5(26)$ & $0(0)$ & $1(9)$ & $2(50)$ & $3(100)$ \\
\hline $\begin{array}{l}\text { Complete response } \\
\text { (CR) }\end{array}$ & $3(16)$ & $0(0)$ & $0(0)$ & $0(0)$ & $0(0)$ \\
\hline Marrow CR & $0(0)$ & $0(0)$ & $0(0)$ & $0(0)$ & $3(100)$ \\
\hline Partial response & $0(0)$ & $0(0)$ & $0(0)$ & $1(25)$ & $0(0)$ \\
\hline Symptom response & $0(0)$ & $0(0)$ & $1(9)$ & $0(0)$ & $0(0)$ \\
\hline Clinical benefit & $2(11)$ & $0(0)$ & $0(0)$ & $1(25)$ & $0(0)$ \\
\hline $\begin{array}{l}\text { Median number of } \\
\text { cycles }\end{array}$ & 5 [2-7] & - & - & 5 [2-7] & $2[1-3]$ \\
\hline $\begin{array}{l}\text { Median number of } \\
\text { cycles to best response }\end{array}$ & $2[1-5]$ & - & - & 3 [2-3] & $1[0-1]$ \\
\hline $\begin{array}{l}\text { Median response } \\
\text { duration } \\
\text { (months) }^{*}\end{array}$ & $2.7[1.9-5.2]$ & - & ${ }^{+} 0.4$ months & $1.9[0-3.8]$ & $2.2[1-2.5]$ \\
\hline No response & $10(53)$ & $5(83)$ & $3(60)$ & $2(50)$ & $0(0)$ \\
\hline Progressive disease & $4(21)$ & $1(17)$ & $1(20)$ & $0(0)$ & $0(0)$ \\
\hline
\end{tabular}

\section{3}

$4 \quad$ *Response duration was censored to the date of transplant in patients who underwent allogeneic SCT.

$5 \quad+$ : response duration corresponding to a single patient who responded to therapy. 
1 Table 3. Univariate and multivariate analysis for survival based on baseline clinicopathologic

2 features.

\begin{tabular}{|c|c|c|c|c|c|c|}
\hline & \multicolumn{3}{|c|}{ UVA } & \multicolumn{3}{|c|}{ Backward MVA } \\
\hline & P-value & HR & $95 \% \mathrm{Cl}$ & $P$ value & HR & $95 \% \mathrm{Cl}$ \\
\hline Age & 0.176 & 1.029 & $0.987-1.073$ & 0.005 & 1.076 & $1.023-1.133$ \\
\hline Female & 0.800 & 0.911 & $0.444-1.872$ & & & \\
\hline $\mathrm{PS} \geq 2$ & 0.059 & 2.053 & $0.973-4.332$ & & & \\
\hline Prior malig. & 0.534 & 1.319 & $0.551-3.153$ & & & \\
\hline Prior chemo/XRT & 0.666 & 1.299 & $0.396-4.259$ & & & \\
\hline Prior transfusion & 0.551 & 1.233 & $0.620-2.452$ & & & \\
\hline Splenomegaly & 0.761 & 1.108 & $0.574-2.139$ & & & \\
\hline B symptoms & 0.483 & 1.290 & $0.633-2.630$ & & & \\
\hline TLS & 0.876 & 1.087 & $0.383-3.082$ & & & \\
\hline WBC & 0.282 & 1.002 & 0.999-1.005 & & & \\
\hline Neu\% & 0.498 & 0.994 & $0.977-1.011$ & & & \\
\hline Blasts \% & 0.192 & 1.051 & $0.975-1.132$ & & & \\
\hline Mono\% & 0.451 & 0.962 & $0.871-1.063$ & & & \\
\hline Lymph\% & 0.223 & 0.975 & $0.935-1.016$ & & & \\
\hline Baso $\%$ & 0.860 & 1.027 & $0.762-1.385$ & & & \\
\hline Eo\% & 0.076 & 0.849 & $0.708-1.018$ & & & \\
\hline $\mathrm{Hgb}$ & 0.033 & 0.846 & $0.726-0.987$ & & & \\
\hline Plt & 0.156 & 0.998 & $0.995-1.001$ & 0.002 & 0.993 & $0.989-0.997$ \\
\hline BM blasts & 0.013 & 1.089 & 1.018-1.165 & $<0.001$ & 1.338 & $1.182-1.515$ \\
\hline BM Eo & 0.339 & 0.932 & $0.807-1.077$ & & & \\
\hline BM baso & 0.935 & 1.005 & $0.884-1.143$ & & & \\
\hline BM mono & 0.027 & 1.172 & $1.018-1.350$ & & & \\
\hline EPO & 0.804 & 1.000 & $0.999-1.001$ & & & \\
\hline$\overline{\mathrm{LDH}}$ & 0.065 & 1.000 & $1.000-1.000$ & $<0.001$ & 1.000 & $1.000-1.000$ \\
\hline UA & 0.435 & 1.042 & $0.939-1.157$ & & & \\
\hline Diploid & 0.130 & 0.607 & $0.318-1.158$ & & & \\
\hline
\end{tabular}




\section{FIGURE LEGENDS}

2 Figure 1. Mutational and clonal landscape of aCML. A. Frequency of identified mutations. Number

3 above each specific gene column represents number of patients sequenced for each specific gene. B.

4 Median and range of variant allele frequencies (VAFs) of mutations identified in at least $10 \%$ of patients.

5 Mutations are ordered by decreasing median VAF. C. Frequency of mutations appearing as dominant or

6 minor events. VAF estimates were used to evaluate clonal relationships within each individual sample

7 using Pearson goodness of fit tests and VAF differences. Clones with the highest VAF or with VAFs close

8 to $40 \%$ were defined as dominant, and those present at VAF $<20 \%$ in the presence of another dominant

9 clone were defined as minor.

11 Figure 2. Clonal changes at the time of leukemic transformation. A. Frequencies of recurrent somatic

12 mutations and cytogenetic abnormalities identified at the time of leukemic transformation that were not

13 present at diagnosis of aCML. B. Dynamic changes in identified somatic mutations and their VAFs from

14 the time of aCML diagnosis to AML.

15

16 Figure 3. Survival outcomes of patients with aCML. A. Kaplan-Meier estimate curve for overall

17 survival of patients with aCML. B. Kaplan-Meier estimate curves for overall survival based on type of

18 therapeutic modality. Other includes hydroxyurea or single agent ruxolitinib. C. Kaplan-Meier estimate 19 curve for leukemia-free survival of patients with aCML.

21 Figure 4. Multivariate Cox proportional hazards model and nomogram for overall survival.

22 Nomogram used by totaling points identified at top scale for each of the independent variables. This

23 summed point score was then identified on a total point scale to identify the 1-year and 3-year survival 24 probabilities. 


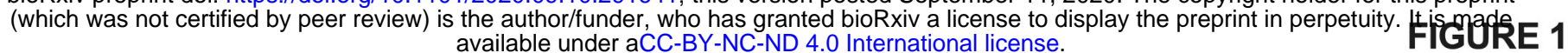

A.

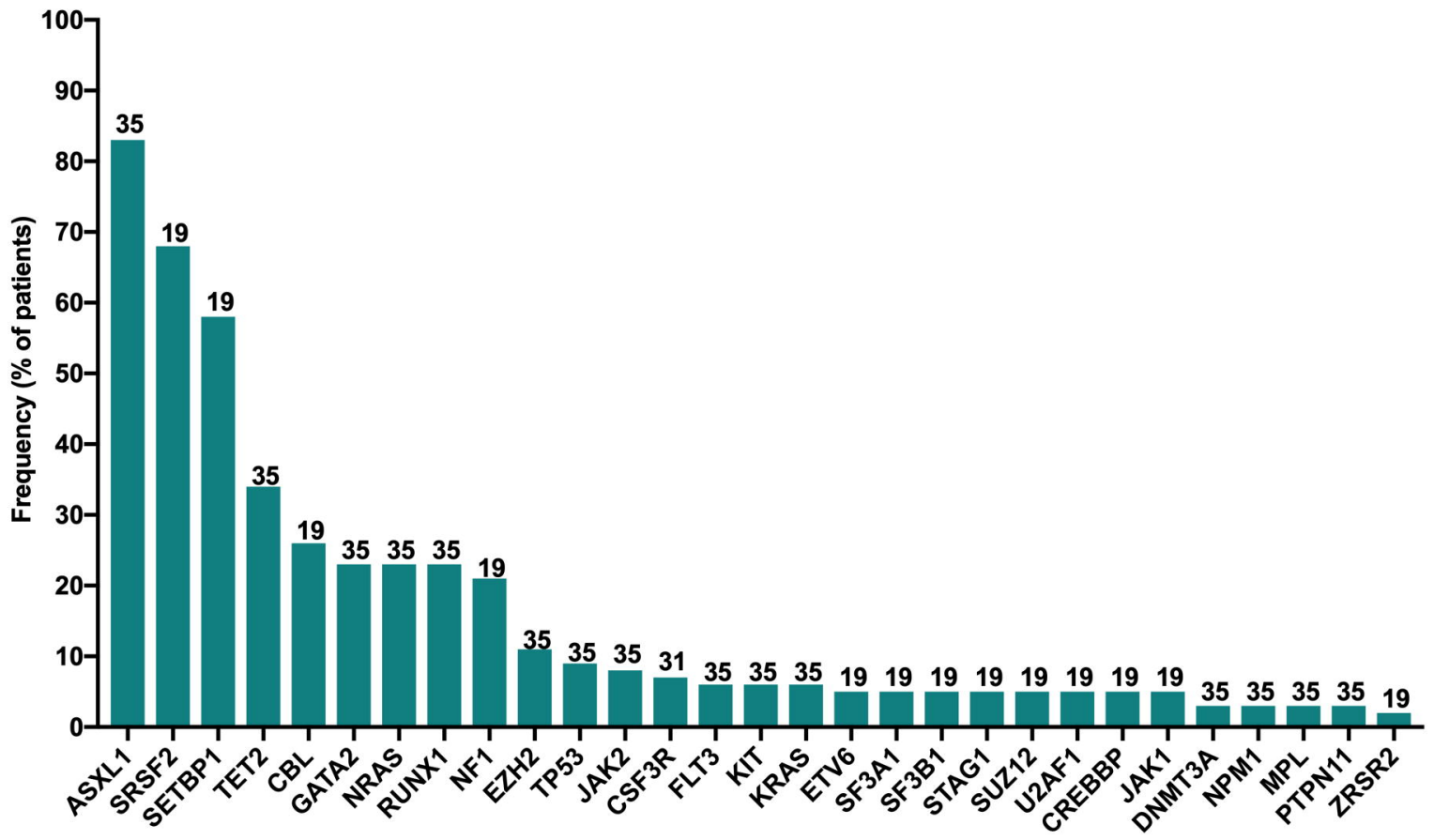

B.

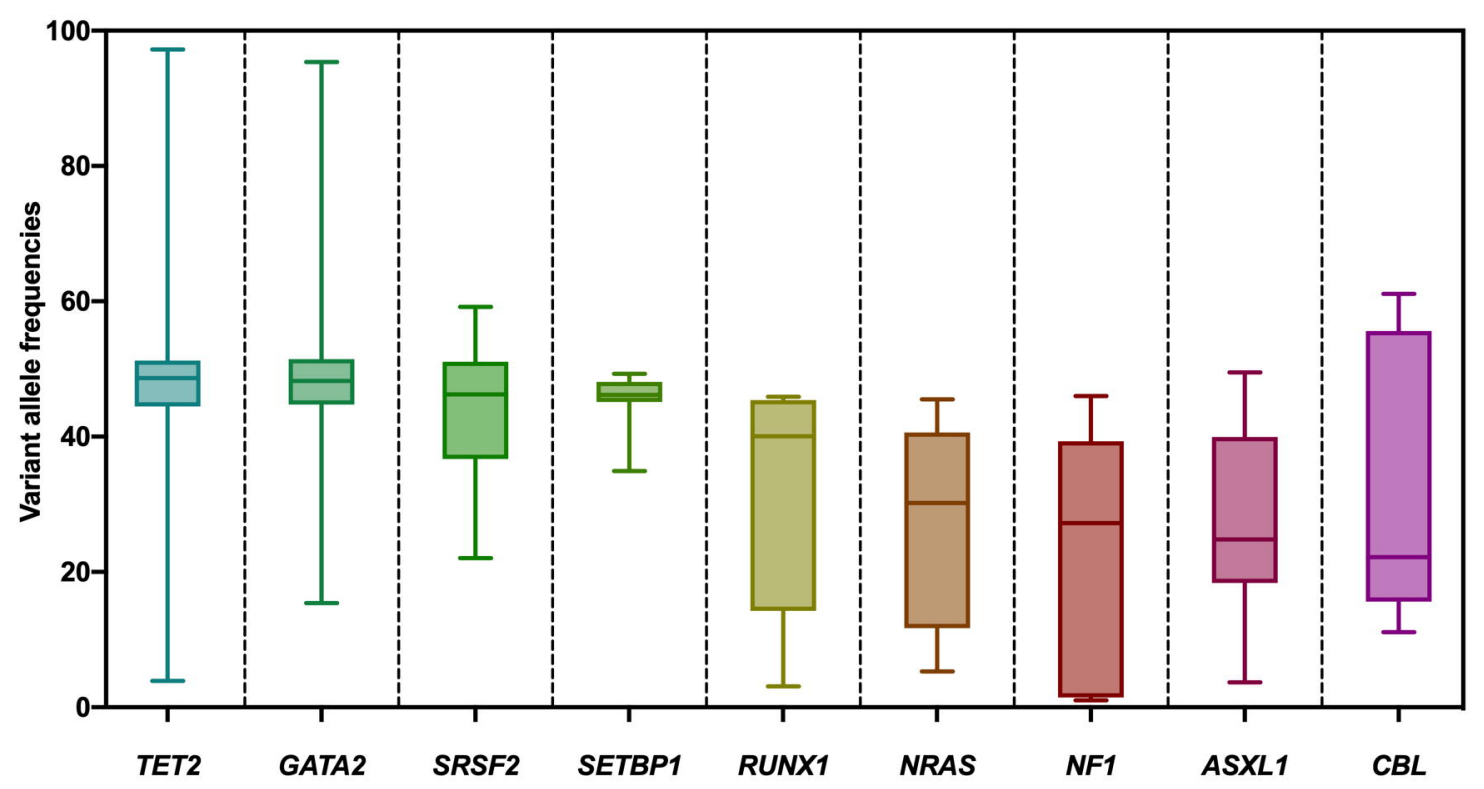

C.

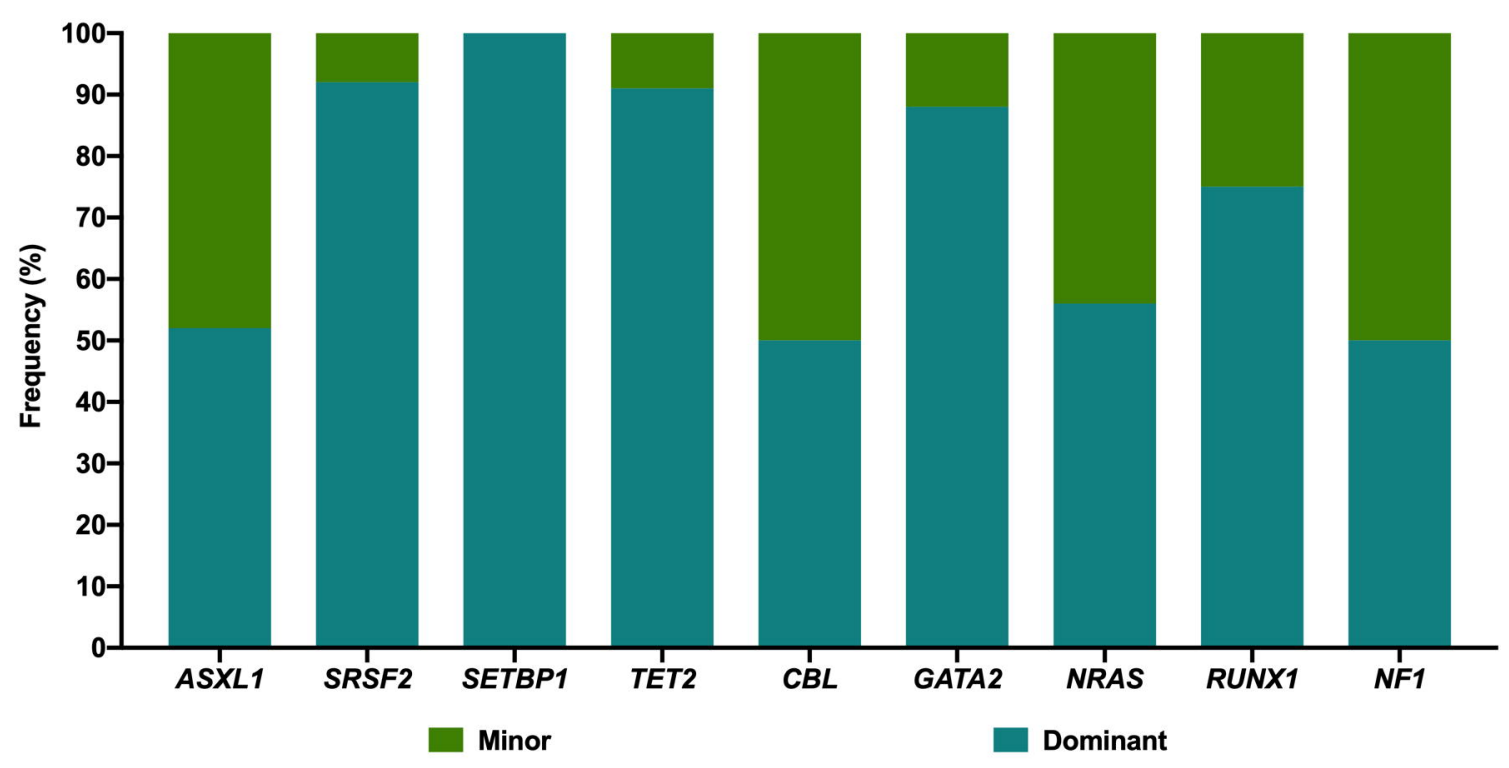




\section{Points}

Age (years)

\section{Platelets (x109/L)}

BM Blast (\%)

\section{LDH (U/L)}

Total points

\section{1-year survival}

3-year survival
0

10

20

30

40

50

60

70

80

90
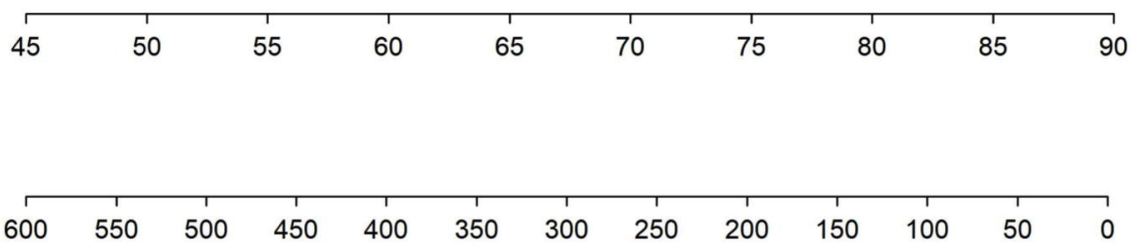

\begin{tabular}{lllllllll}
\hline 0 & 2 & 4 & 1 & 1 & 1 & 14 & 16
\end{tabular}

$5000 \quad 10000$

15000

20000

25000

30000

35000

40000

\begin{tabular}{|c|c|c|c|c|c|c|c|c|c|}
\hline \multicolumn{10}{|c|}{ Points } \\
\hline 0 & 20 & 40 & 60 & 80 & 100 & 120 & 140 & 160 & 180 \\
\hline
\end{tabular}

0.99

0.95

$\begin{array}{llllllll}0.7 & 0.6 & 0.5 & 0.4 & 0.3 & 0.2 & 0.1\end{array}$

0.01 